\title{
Toxicity of Silver Nanoparticles to Human Cell Lines
}

\section{Singh Z*}

P G Department of Zoology, Khalsa College Amritsar, India

*Corresponding author: Zorawar Singh, P G Department of Zoology, Khalsa College Amritsar, Punjab, India, PIN: 143001, Tel: +91-9417230075; Email:

Short Communication

Volume 3 Issue 4

Received Date: December 08, 2018

Published Date: December 27, 2018

DOI: $10.23880 /$ nnoa- 16000154 zorawarsinghs@rediffmail.com

\section{Abstract}

Antibacterial effect of silver nanoparticles (SNPs) has resulted in their widespread applications in health, electronics, medicine and home products. SNPs also possess unique optic and catalytic properties, making them highly attractive for the creation of new advanced functional materials. Once released into the environment, SNPs may change chemically and pose toxicity to different faunal species including humans. SNPs may penetrate the gastrointestinal barrier and reach the circulatory system resulting in severe health impacts. The present review paper emphasizes the toxicity of SNPs on different human cell lines including human adipose-derived stem cells, human hepatoma cells, human mesenchymal stem cells, human intestinal cells, human epidermoid larynx carcinoma cell line and human Jurkat T cells through compiling the available reports. SNPs were found to display toxicity to different cell lines at different concentrations. SNP exposure to humans should be minimized so as to minimize the health hazards.

Keywords: Silver Nanoparticles; Toxicity; Human; Cell Lines; HepG2; Oxidative Stress

\section{Introduction}

In recent years, there has been a growing interest in the biomedical applications of nanoparticles. In particular, silver nanoparticles (SNPs) are increasingly being investigated as tools for novel cancer therapeutics, capitalizing on their unique properties to enhance potential therapeutic efficacy. SNPs have been classified as the most abundant nanoparticles found in commercial products. However, questions as to are we able to contain or control the toxicity effects of SNPs, and how much do we know about the toxicological profile of SNPs which are commonly used in emerging nanotechnology-based applications, still remain unanswered [1].
The antibacterial effect of silver nanoparticles has resulted in their extensive application in health, electronics and medicine. However, SNPs remain a controversial area of research with respect to their toxicity in biological and ecological systems [2]. SNPs also possess unique optic and catalytic features, making them highly interesting for the creation of novel and advanced functional materials [3]. As a result of the extensive number of applications of SNPs, their potential impacts, once released into the environment, are of concern. SNPs readily transform in the environment, which modifies their properties and alters their transport, fate, and toxicity. It is essential to consider such transformations when assessing the potential environmental impact of SNPs [4]. The toxicity of SNPs was reported to be 


\section{Nanomedicine \& Nanotechnology Open Access}

dependent on various factors such as particle size, shape and capping agents [5]. Surface charge is one of the most important factors that govern the toxicity of SNPs. It is increasingly used in a variety of medical and consumer products resulting in increased exposure. However, the knowledge on the systemic toxicity of nanosilver is relatively limited [6]. The major toxicological concern associated with nano-moieties is the fact that some manufactured nanomaterials are redox active, and some particles transport across cell membranes, especially into mitochondria [7]. Several experiments have demonstrated that SNPs can be toxic to the vital organs of humans, especially to lungs [8]. Orally ingested nanoparticles may overcome the gastrointestinal barrier, reach the circulatory system, be distributed in the organism, and cause adverse health effects [9]. Capping agents may also affect the properties of SNPs. A study demonstrated how the concentration of the capping agent plays a major role in determining the dimensions, morphology, and stability, as well as toxicity of a silver colloidal solution [10].

SNPs are the most frequently commercialized nanomaterial currently [11]. Due to a distinct lack of information on hazardous properties of SNPs on humans, there is a dire need to conduct experimental studies which can reveal the actual health impact of these nanomoieties. With the same aim, the present review emphasizes on the toxic properties of SNPs as reported by different studies conducted on human cell lines.

\section{Studies Based on Human Cell Lines}

SNPs have been employed in a number of studies to assess their toxic profile. Kawata et al. [12] evaluated invitro toxicity of SNPs at non-cytotoxic doses to HepG2 human hepatoma cells based on cell viability assay, micronucleus test and DNA microarray analysis. Both nano-sized $\mathrm{Ag}$ as well as ionic $\mathrm{Ag}^{+}$contributed to toxic effect of SNPs by causing cell proliferation at low doses (< $0.5 \mathrm{mg} / \mathrm{L}$ ). The experiments exhibited cytotoxicity at higher doses (> 1.0mg/L) and abnormal cellular morphology, displaying cellular shrinkage and acquisition of an irregular space. Exposure to SNPs also increased the frequency of micronucleus formation causing much stronger damage to chromosomes. Similarly, Kim et al. [13] studied the oxidative stress-dependent toxicity of silver nanoparticles in human hepatoma cells and results revealed that cytotoxicity is primarily the result of oxidative stress and is independent of toxicity of $\mathrm{Ag}^{+}$ion. In another study, DNA damage, toxicity and functional impairment in human mesenchymal stem cells (hMSCs) on SNP exposures was evaluated. The study demonstrated cyto- and genotoxic potential of SNPs in hMSCs at significantly higher concentrations as compared to antimicrobial effective levels [11]. Bohmert, et al. [9] studied the analytically monitored digestion of SNPs and their toxicity in human intestinal cells. SNPs may overcome the gastrointestinal juices in their particulate form without forming large quantity of aggregates. These particles can reach the intestinal epithelial cells after ingestion with only a slight reduction in their cytotoxic potential. The effect of synthesized SNPs from the leaf of Suaeda monoica on Human Epidermoid Larynx Carcinoma cell line was evaluated by the MTT colorimetric technique. Results showed a dose-dependent toxicity for the cell tested and the viability of Hep-2 cells decreased to $50 \%$ $\{\mathrm{IC}(50)\}$ at the concentration of $500 \mathrm{nM}$ [14].

A comprehensive toxicity assay was conducted on human Jurkat $\mathrm{T}$ cells using oxidative stress-related endpoint. The effect of Ag ions was also investigated. Cell viability tests indicated high sensitivity of Jurkat $\mathrm{T}$ cells when exposed to SNPs compared to Ag ions; however, both SNPs and Ag ions induced similar levels of cellular reactive oxygen species during the initial exposure period and; after $24 \mathrm{~h}$, they were increased on exposure to SNPs compared to Ag ions, which suggest that oxidative stress may be an indirect cause of the observed cytotoxicity of SNPs. SNPs exposure activates p38 mitogen-activated protein kinase through nuclear factor-E2-related factor-2 and nuclear factor-kappa B signaling pathways, subsequently inducing DNA damage, cell cycle arrest and apoptosis [15].

On the contrary, some studies have found a nonsignificant effect of SNPs as Samberg et al. [16] evaluated the toxicity and cellular uptake of both undifferentiated and differentiated human adipose-derived stem cells (hASCs) exposed to SNPs and assessed their effect on hASC differentiation. Exposure of hASC to either 10- or 20-nm SNPs resulted in a non-significant cytotoxicity to hASC. Each of the hASC, adipogenic and osteogenic cells showed cellular uptake of both 10- and 20-nm SNPs, without causing significant ultrastructural alterations. Moreover, exposure did not influence the differentiation of the cells [16].

\section{Conclusion}

Silver nanoparticles (SNPs) have been found as the most abundant nanoparticles used in commercial products. The exposure of humans to SNPs may result in severe health hazards. Reviewed studies reveal SNPs to 


\section{Nanomedicine \& Nanotechnology Open Access}

cause toxic effects in human cell lines including human adipose-derived stem cells, human hepatoma cells, human mesenchymal stem cells, human intestinal cells, human epidermoid larynx carcinoma cell line and human Jurkat $\mathrm{T}$ cells. Conclusively, exposure to SNPs should be minimized to lower the chances of its deleterious health impacts.

\section{Funding}

There is no funding source for the present study

\section{References}

1. Ong C, Lim JZ, Ng CT, Li JJ, Yung LY, et al. (2013) Silver nanoparticles in cancer: therapeutic efficacy and toxicity. Curr Med Chem 20(6): 772-781.

2. Kim YS, Song MY, Park JD, Song KS, Ryu HR, et al. (2010) Subchronic oral toxicity of silver nanoparticles. Part FibreToxicol 7: 20.

3. Haase A, Mantion A, Graf P, Plendl J, Thuenemann AF, et al. (2012) A novel type of silver nanoparticles and their advantages in toxicity testing in cell culture systems . ArchToxicol 86(7): 1089-1098.

4. Levard C, Hotze EM, Lowry GV, Brown GE (2012) Environmental transformations of silver nanoparticles: impact on stability and toxicity. Environ Sci Technol 46(13): 6900-6914.

5. El Badawy AM, Silva RG, Morris B, Scheckel KG, Suidan MT, et al. (2011) Surface charge-dependent toxicity of silver nanoparticles. Environ Sci Technol 45(1): 283-287.

6. De Jong WH, Van Der Ven LT, Sleijffers A, Park M V, Jansen EH, et al. (2013) Systemic and immunotoxicity of silver nanoparticles in an intravenous 28 days repeated dose toxicity study in rats. Biomater 34 : 8333-8343.

7. Teodoro JS, Simoes AM, Duarte F V, Rolo AP, Murdoch RC, et al. (2011) Assessment of the toxicity of silver nanoparticles in vitro: a mitochondrial perspective. Toxicol In Vitr 25(3): 664-670.

8. Chairuangkitti P, Lawanprasert S, Roytrakul S, Aueviriyavit S, Phummiratch D, et al. (2013) Silver nanoparticles induce toxicity in A549 cells via ROSdependent and ROS-independent pathways. Toxicol In Vitr 27(1): 330-338.

9. Böhmert L, Girod M, Hansen U, Maul R, Knappe P, et al. (2014) Analytically monitored digestion of silver nanoparticles and their toxicity on human intestinal cells. Nanotoxicology 8(6): 631-642.

10. Stevanovic M, Kovacevic B, Petkovic J, Filipic M, Uskokovic D (2011) Effect of poly-alpha, gamma, Lglutamic acid as a capping agent on morphology and oxidative stress-dependent toxicity of silver nanoparticles . Int J Nanomedicine 6: 2837-2847.

11. Hackenberg S, Scherzed A, Kessler M, Hummel S, Technau A, et al. (2011) Silver nanoparticles: evaluation of DNA damage, toxicity and functional impairment in human mesenchymal stem cells. Toxicol Lett 201(1): 27-33.

12. Kawata K, Osawa M, Okabe S (2009) In vitro toxicity of silver nanoparticles at noncytotoxic doses to HepG2 human hepatoma cells. Environ Sci Technol 43(15): 6046-6051.

13. Kim S, Choi JE, Choi J, Chung KH, Park K, et al. (2009) Oxidative stress-dependent toxicity of silver nanoparticles in human hepatoma cells. Toxicol In Vitr 23: 1076-1084.

14. Satyavani K, Gurudeeban S, Ramanathan T, Balasubramanian T (2012) Toxicity Study of Silver Nanoparticles Synthesized from Suaeda monoica on Hep-2 Cell Line. Avicenna J Med Biotechnol 4(1): 3539.

15. Eom HJ, Choi J (2010) p38 MAPK activation, DNA damage, cell cycle arrest and apoptosis as mechanisms of toxicity of silver nanoparticles in Jurkat T cells. Environ Sci Technol 44(21): 83378342.

16. Samberg ME, Loboa EG, Oldenburg SJ, MonteiroRiviere NA (2012) Silver nanoparticles do not influence stem cell differentiation but cause minimal toxicity. Nanomedicine(Lond) 7: 1197-1209. 